\title{
Aircraft safety and operational efficiency during LGS operations at the Large Binocular Telescope Observatory.
}

\author{
Gustavo Rahmer $^{* a}$, Michael Lefebvre ${ }^{\mathrm{a}}$, Julian C. Christou ${ }^{\mathrm{a}}$ \\ ${ }^{a}$ Large Binocular Telescope Observatory, 933 North Cherry Avenue, Tucson, AZ 85721 USA
}

\begin{abstract}
A key aspect of LGS (Laser Guide Star) operations is the implementation of measures to prevent the illumination of aircraft flying overhead.

In parallel to the use of aircraft spotters, we have an automatic aircraft detection system developed at UCSD known as TBAD (Transponder-Based Aircraft Detection).

Our airspace is busier, if not the busiest, than for other telescope sites using LGS, and the constant interruptions, specially during the first half of the night, can have a big impact in the efficiency of the LGS operations.

We describe the steps that we have taken to validate the operation of the automatic detection system, and show that the efficiency of our laser operation will improve by transitioning to a TBAD only-operation.
\end{abstract}

Keywords: Laser Guide Star, Ground-Layer Adaptive Optics, observatory operations, automatic aircraft detection.

\section{INTRODUCTION}

ARGOS (Advanced Rayleigh-guided Ground layer adaptive Optics System) [1] is the multi-beacon LGS system for the Large Binocular Telescope Observatory, designed to be used with the LUCI instruments: a pair of NIR imagers and multi-object spectrographs.

The system projects three laser beams from the back of each of the two secondary mirror units, which create two constellations circumscribed on circles of 2 arcmin radius with 120 degree spacing. Each of the six Nd:YAG lasers provides a beam of green $(532 \mathrm{~nm})$ pulses at a rate of $10 \mathrm{kHz}$ with a power of $14 \mathrm{~W}$ to $18 \mathrm{~W}$ each, for a combined power of approximately $50 \mathrm{~W}$ per side [1].

The propagation of Class 4 lasers towards the sky constitutes a serious hazard for aircraft. The high-powered, low divergence beams from ARGOS result in an extremely large nominal ocular hazard distance (NOHD) extending well into the airspace of commercial aircraft. Hence, the potential for acute laser eye damage exists if a direct beam or specular reflection were to enter the aircraft cockpit. More likely however, diffuse laser beams could interfere with the vision of the pilot or air crewmember causing temporary flashblindness, glare and disruption or distraction and startle when the laser beam illuminates the windshield of an aircraft [2].

The Federal Aviation Administration (FAA) has the authority to regulate the safe and efficient use of the navigable airspace, and to that end it requires that laser operators follow a process of registration and notification of planned laser operations.

The corresponding documentation, which includes information about the lasers and the planned mitigation plans (human spotters, automatic aircraft detection), was submitted in April 2012. Approval for operation was received on June 2012. Our "first light" took place in November 2013.

The approval document, referred by the FAA as "Letter of Determination", specifies the conditions to be followed for the lawful operation of the lasers. In particular, our operation is currently limited to a minimum elevation of $45^{\circ}$, and we are required to have visual aircraft spotters stationed outside the telescope with complete view of the affected airspace.

*grahmer@lbto.org; phone 1-520-626-7152 
To ensure full coverage of the affected airspace, as required by the FAA based on relevant ANSI standards [3], two spotter stations were selected outside the telescope building, on opposite sides. One is located to the southeast of the building, close to the main entrance, and the other is to the northwest of the building, close to a secondary entrance.

Most of the air traffic is east-west, so these locations are well-suited for a proper monitoring of the traffic, and also have the added benefit of minimizing the travel distance from the inside of the building to the station during shift changes.

Figure 1 shows a satellite view of the Mount Graham International Observatory (MGIO) site with the location of the stations. North is up and East is to the right.

It should be noted also that we had an extra restriction on selecting the location of the stations. Due to National Forest Service rules, we were limited by a defined boundary around the building.

Also, the installation of permanent structures outside the building is not allowed. In a site where low temperatures and wind chill are major concerns, we have been looking for ways to protect the spotters from the elements without impairing their sky coverage. To protect the spotters from the wind, we found that the simplest and most effective solution was to park a truck in an orientation such that it can be used to block the wind. A comfortable reclining chair is provided in place.

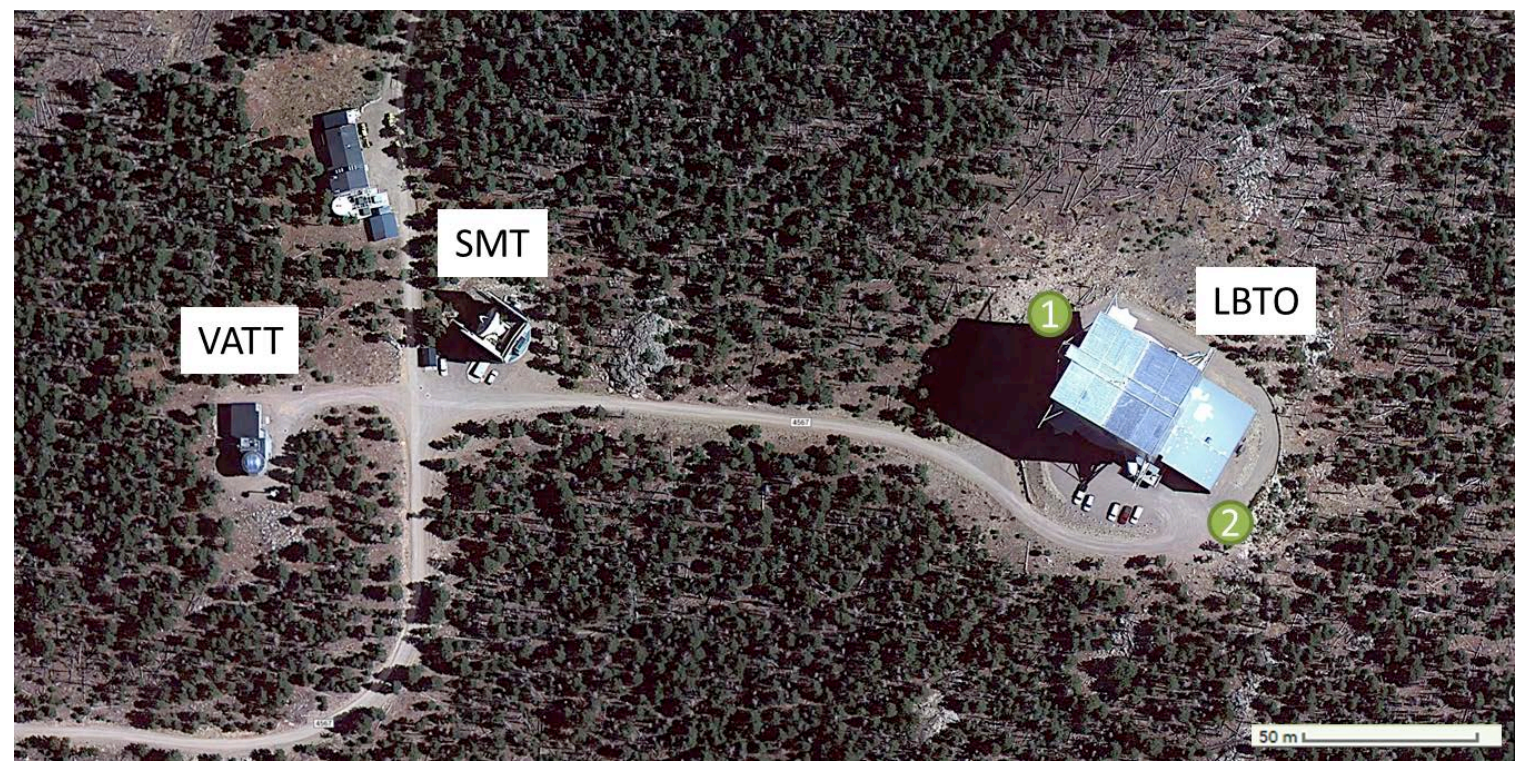

Figure 1: The MGIO site with the three telescopes and the locations of the aircraft spotter stations for LBTO: Station 1 is on the northwest side and Station 2 is on the southeast side. North is up.

Our standard scheme uses four spotters, two on station and two standby, and shifts of 45 minutes to one hour, depending on the weather conditions, resulting on breaks that last the same time than a shift.

Air traffic in the vicinity of Mount Graham is quite intense, consisting mostly of east-west transcontinental flights and flights approaching the two major nearby airports, Phoenix and Tucson, from the east. During our laser propagation campaigns, we have observed that the commercial air traffic decreases significantly after midnight, local time.

Closures due to aircrafts depend on several factors. Objective factors are: telescope pointing, time that lasers are being propagated, and air traffic density.

Unfortunately the spotters add a subjective dimension to the operation. Their perception of aircraft awareness is affected by trying to build a mental 3-D space based on basically a 2-D setup at night. Furthermore, their visual sensitivity and accuracy may be impacted by the presence of moonlight. Our laser propagation campaigns are usually scheduled around full moon, given that our science camera works in the NIR.

This makes the need of an automatic aircraft detection system a must. 


\section{AUTOMATIC AIRCRAFT DETECTION}

\subsection{Background}

The ARGOS project included an automatic aircraft detection system based on visible and IR cameras. The system did not work as expected, so we decided to take a different path for aircraft detection: the TBAD (Transponder Based Aircraft Detection) system developed at UCSD [4].

The basic TBAD concept is the implementation of two antennas (built on the same physical substrate) aligned with the optical axis of the telescope, one with a beam width (full width at half-power) of about $24^{\circ}$ (settable) and the other with a beam width of about $90^{\circ}$ ("omni"). The ratio of the power received by the narrow-beam antenna to that received by the broad-beam antenna depends only on the angular position of the transponder with respect to the beam axis. In particular, it does not depend on the distance, transmitted power, or polarization mismatch. The detection signal from each system is connected to the ARGOS Interlock System to stop propagation automatically when any of them is triggered.

The use of two systems in parallel provides not only better coverage, but also redundancy.

Due to the unique configuration of the LBT (two laser launch systems through separate enclosure slits), our approach is to have independent TBAD systems on each side. The ideal location for the antennas is behind the secondary mirror, but in our case that area is completely taken by the laser launch mirror LM2, and therefore we decided to mount the antennas at the end of an arm mounted at the top of the telescope structure and as close to the projection of the edge of the mirror without creating any obscuration.

The unobstructed field of view (FoV) of the antennas in the horizontal direction will be constrained by the distance to the enclosure slits and their width when fully open. It will vary from a maximum of $70.5^{\circ}$ when pointing to zenith to a minimum of $37.8^{\circ}$ at an elevation of $51.5^{\circ}$, as shown in Figure 4. The minimum FoV is still bigger than the narrow-beam aperture, and therefore does not diminish the performance of the system.

TBAD systems have been in operation at Apache Point Observatory (APO) since 2009 [6], and at W. M. Keck Observatory (WMKO) since 2012 [5]. For both observatories the FAA has approved the use of TBAD without human spotters, which is our ultimate goal for an efficient operation.

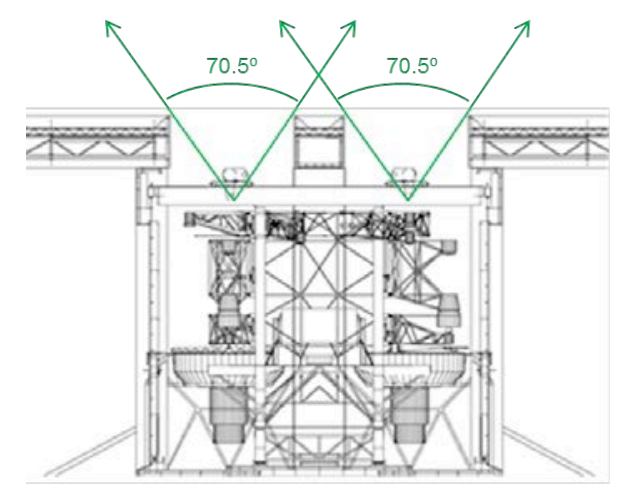

Max FoV at Zenith

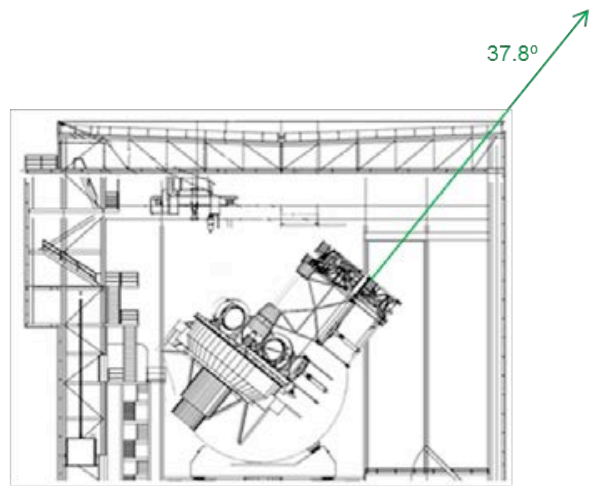

Min FoV at Elevation $51.5^{0}$

Figure 2: Maximum and minimum unobstructed horizontal Field of View for the TBAD antennas. 


\subsection{System installation}

The TBAD systems were installed on the telescope on April 2015. Both systems had the standard narrow beam setting of $24^{\circ}$. As mentioned above, the location chosen for the antennas was the center of the M2 swingarm, outside the footprint of the primary mirror to avoid any obscuration, as shown in Figure 3.

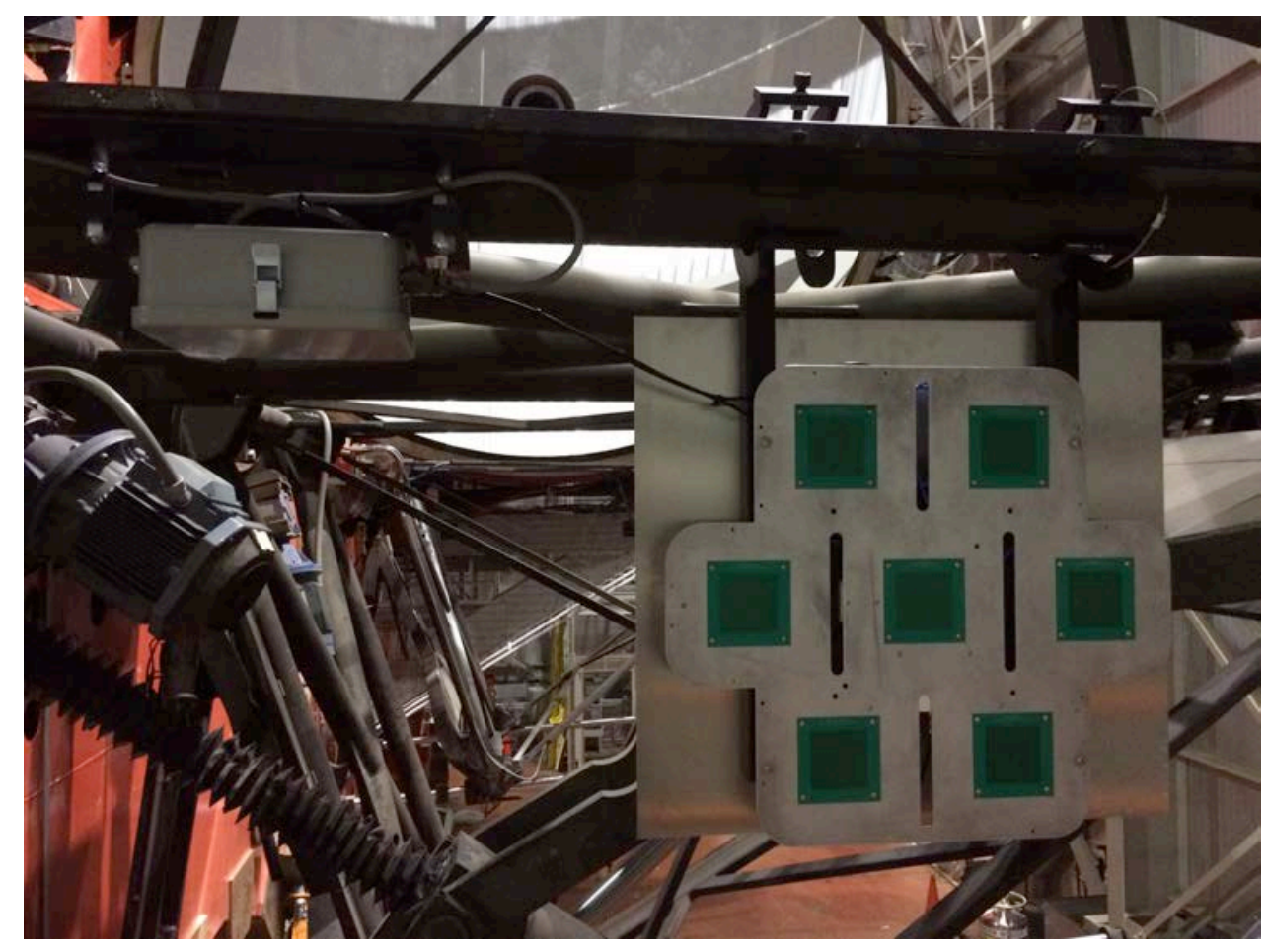

Figure 3: One of the TBAD antennas mounted on the SX (Left) M2 swingarm. The primary mirror is seen in the background.

\subsection{Data collection}

TBAD provides a way to log the transponder data being received, which is independent of the triggering of the shutter signal, through a serial connection.

A Python script listening to the corresponding serial port is able to provide a log of either the "in-beam" codes or the "omni" codes (i.e. all the chatter received).

In our case we use the "omni" option to collect as much data as possible for analysis.

For every code received, the Python script provides a basic decodification, and also includes telescope status (azimuth, elevation and state of the enclosure doors: open or close).

We activate this script automatically for every night of operations, even if there is no laser propagation. Problems with the script prevented us from collecting useful data during the first months of TBAD operation. Reliable data started to be collected in September 2015 (after the Telescope Summer Shutdown). 


\section{DATA ANALYSIS}

\subsection{TBAD detection statistics}

As described in [7], the number of TBAD detections per night is over 6 , with most of the detections taking place during the first half of the night, as shown in the histogram in Figure 4.

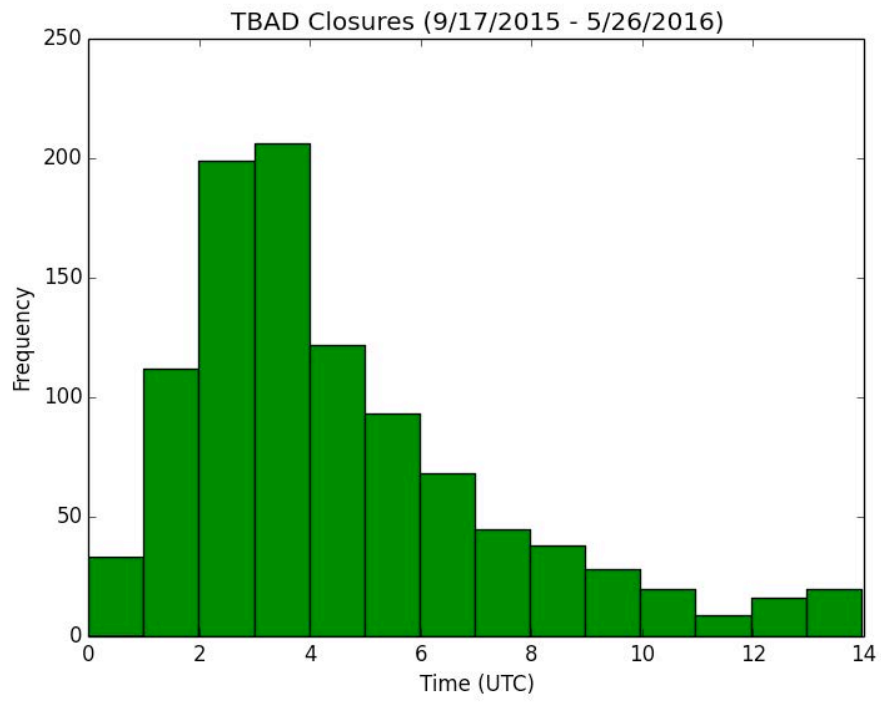

Figure 4: Histogram of TBAD detections with respect to UTC time (Local Time $=$ UTC - 7).

Using the telescope azimuth and elevation data embedded in the TBAD logs it is possible to get a sense of the "preferred" telescope pointing for TBAD detections.

Histograms of azimuth and elevation data for the same period are shown in Figure 5 and 6 respectively

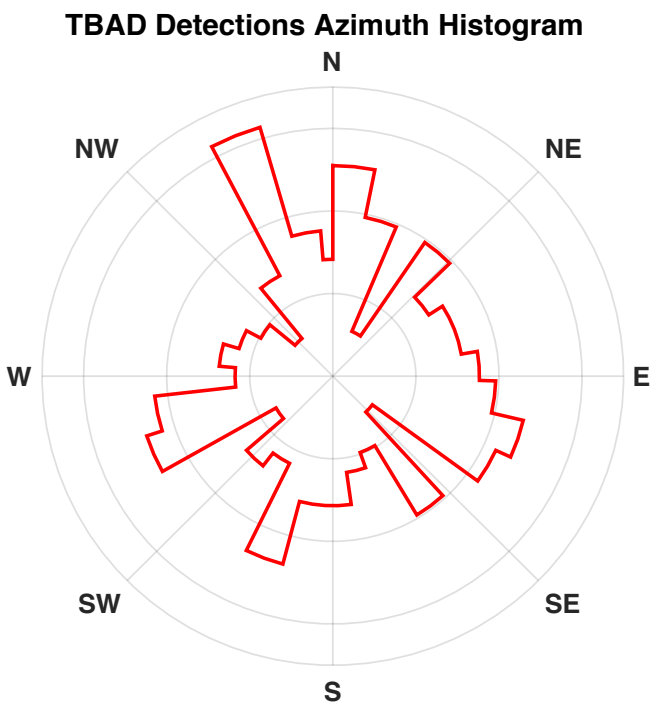

Figure 5: Histogram of TBAD detections with respect to telescope Azimuth. 
TBAD Detections Elevation Histogram

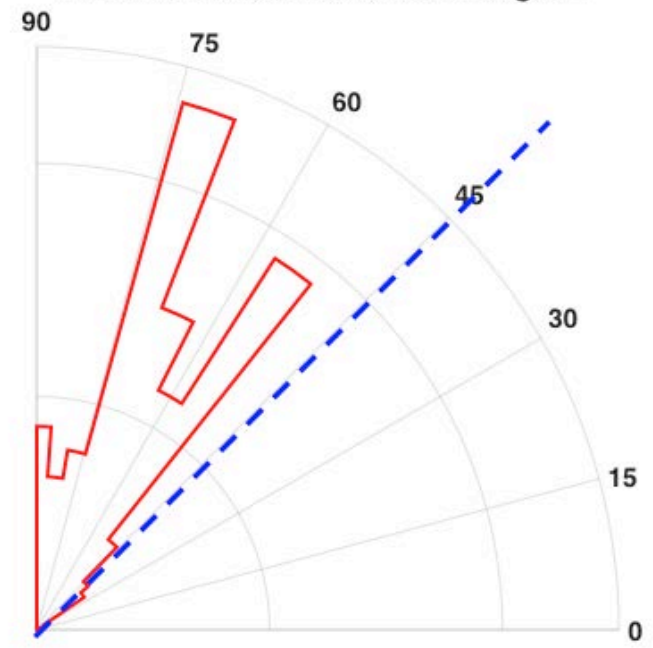

Figure 6: Histogram of TBAD detections with respect to telescope Elevation. The dashed blue line shows the 45 degrees elevation limit for laser propagation imposed by the FAA.

Combining azimuth and elevation data for the last three ARGOS campaigns (October 2016, December 2016, March 2017 and May 2017), with an average of 9 nights each, we can also visualize the spread of coordinates, as shown in Figure 7.

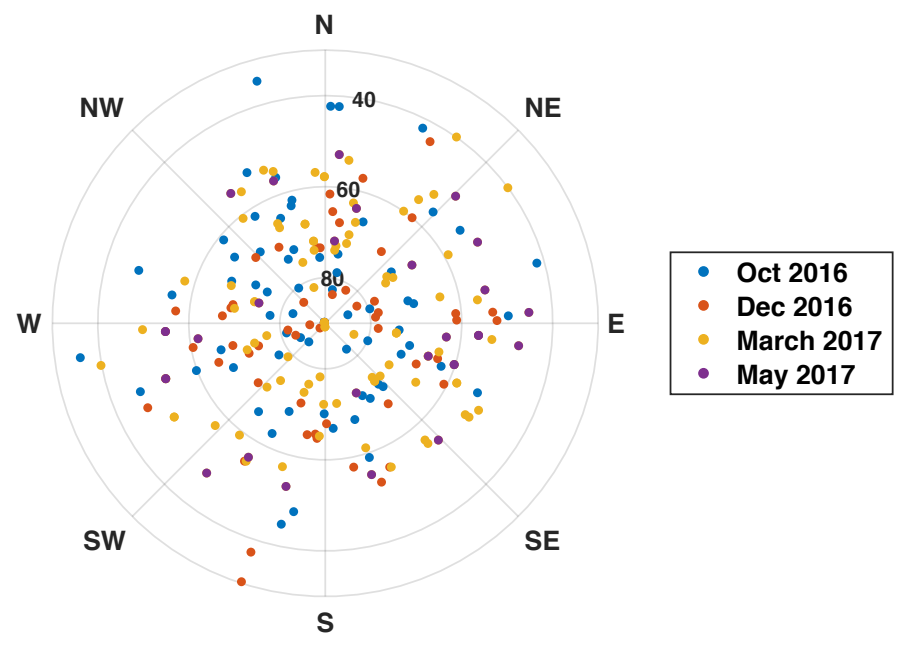

Figure 7: Combined display of TBAD detection coordinates (Azimuth/Elevation) for the last four ARGOS campaigns. 


\subsection{ADS-B data}

ADS-B (Automatic Dependent Surveillance - Broadcast) is a type of data broadcast by airplanes that provide identification, current position, altitude, and velocity information. Although not mandatory, it is used currently by most airplanes and will become mandatory for commercial aviation in the US in 2020 [8].

Our TBAD system is able to decode this "self-reported" data, which provides another way to validate the accuracy of the system.

We can plot the reported position of an airplane with respect to our detection cone, and thus verify that TBAD did detect the airplane when it was supposed to. An example of this type of plot is shown in Figure 8. The circle in the center of the plot defines the nominal 12.5 degrees radius of the detection cone. The dots represent the reported position, and the colors indicate the status of the TBAD detection signal: red for "in-beam" shuttered, yellow for shuttered but not "inbeam" and blue for not shuttered.

The dots should always be either red or yellow inside the cone boundary. Due to the nature of the detection system (ratio of signal levels from the directional antenna to the omni antenna), the edge of the detection cone is not perfectly defined, and therefore it is expected to have "in-beam" events even if the reported location is outside the detection boundary.

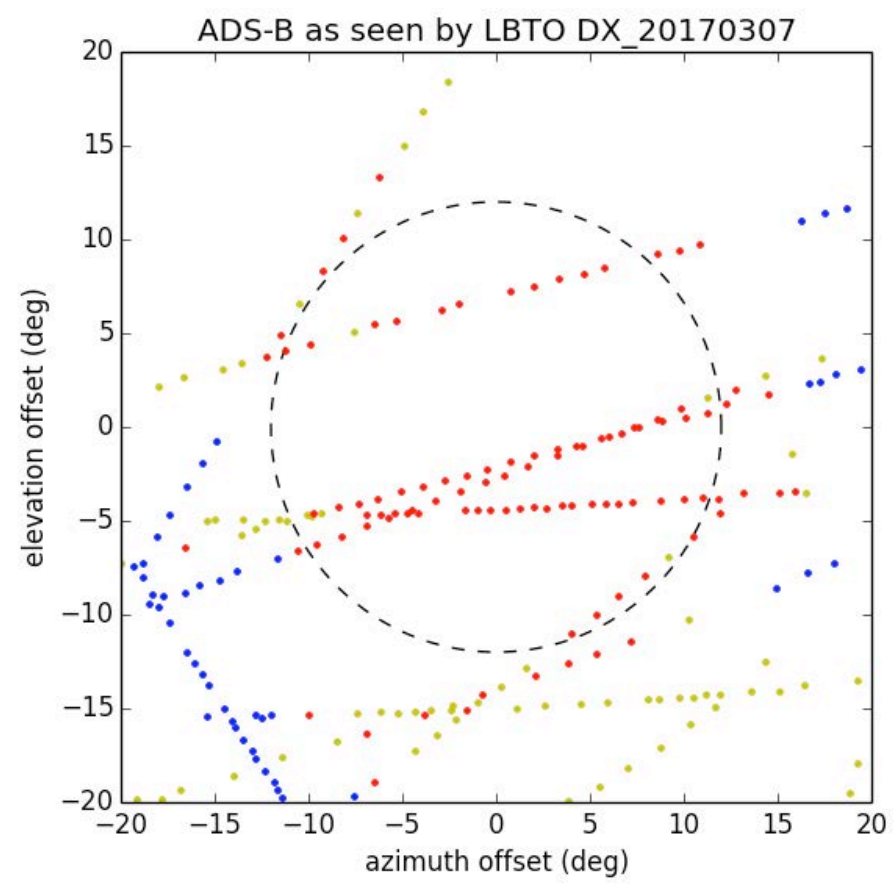

Figure 8: Airplane location with respect to the telescope pointing as calculated from ADS-B data for the night of March $7^{\text {th }}$, 2017. Colors indicate the status of the TBAD signal: red for "in-beam" shuttered, yellow for shuttered but not "in-beam" and blue for not shuttered.

The ADS-B plots are also very useful to provide a sense of the amount of aircraft traffic detected by TBAD. Figure 9 shows a mosaic of the ADS-B plots for the nights of the March 2017 ARGOS campaign.

The plots show that all airplanes that crossed the detection cone boundary were successfully detected. 

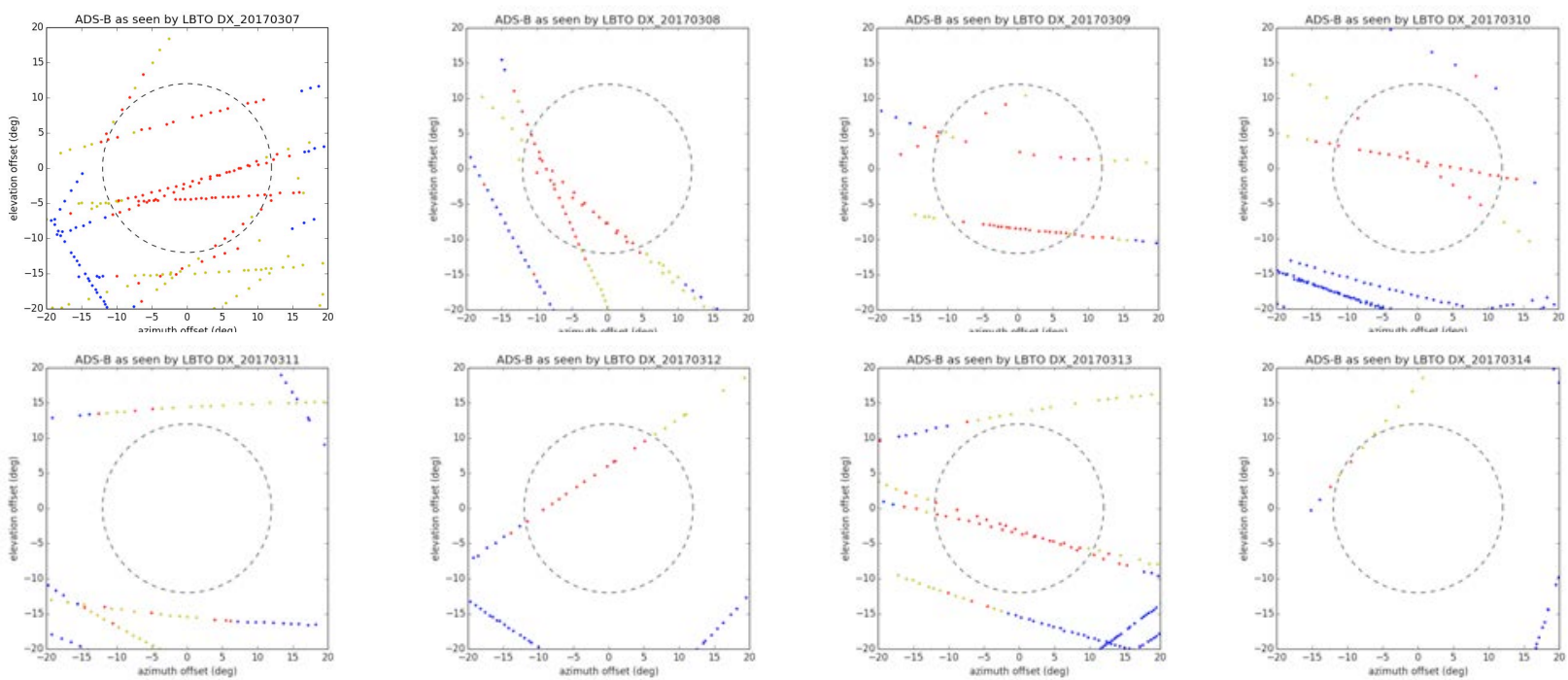

Figure 9: ADS-B plots for the March 2017 ARGOS campaign (March $7^{\text {th }}$ to March $14^{\text {th }}, 2017$ ).

\section{VALIDATION}

As a way to try to cross-check the TBAD detections against an independent source, we purchased a subscription to the commercial product Flight Explorer (FE) from Sabre, Inc.

This software can be customized to generate logs of air traffic in a defined airspace, and has been used successfully at Keck [5] and APO [6] for their own TBAD verification.

We get records for airplanes passing through a $25 \mathrm{NM}$ radius cylinder centered on LBTO and capturing air traffic above $10,000 \mathrm{ft}$ MSL. For each airplane in the region, we get a time stamp, latitude and longitude, altitude, heading, speed, flight number, origin, and destination.

During ARGOS operation, we log all the warnings and alerts from our aircraft spotters, and also use the real-time information provided by the publicly available website flightradar24.com. The website also provides historical information, as it is possible to "go back in time" to revisit previous airplane trajectories in a graphical way.

An example of this 4-way approach to validation (TBAD $\log$, FE $\log$, flightradar24.com display and spotters $\log$ ) is shown in Figure 10. 

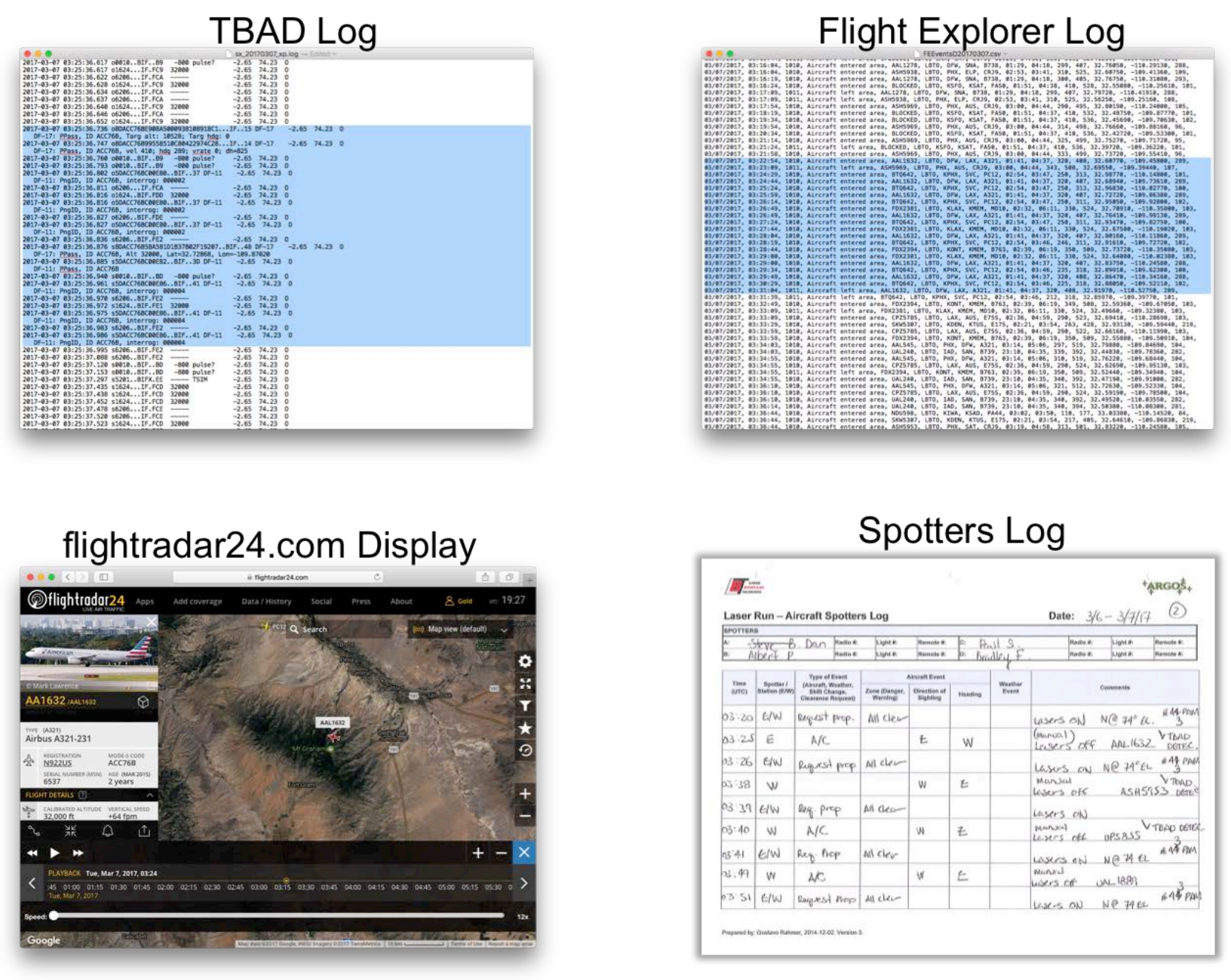

Figure 10: Validation data sources: TBAD log, FE log, flightradar24.com display and spotters log.

Using all this data, we verify that every airplane that entered the detection cone boundary was correctly detected by TBAD.

We do not use ADS-B for official validation, as its use is not mandatory yet (see section 3.2).

We finally compile a summary of TBAD detection validation for every ARGOS campaign. Below we present summary tables for the last two campaigns: March 2017 and May 2017.

In these tables, an "event" is a propagation shutter closure initiated by the laser operator, following a request by the aircraft spotters. Most of these events are also detected by TBAD, but some are "spotter-only", which reflects the inherent subjective nature of the human perception.

These false positives, although not many, certainly have an impact in the efficiency of our operation. For example, during the March campaign they represented $20 \%$ of all events.

Also, given the communication delay between the spotters and the laser operator, they are explicitly asked to be more conservative. Their "danger" zone is actually 25 degrees, compared to TBAD's 12.5 degrees. This increases the closure time for propagation. 
For our validation process, the important numbers for TBAD validation is given by the "\#FE only (no TBAD)" column, which is zero, proving that every airplane that crossed the cone boundary was successfully detected by TBAD.

The numbers in red refer to a mention in the "Comments" section for the corresponding day.

\section{March 2017 Laser Run Summary}

\begin{tabular}{|c|c|c|c|c|c|c|c|}
\hline UTC date & $\begin{array}{l}\text { Telescope } \\
\text { open time } \\
\text { (hours) }\end{array}$ & $\begin{array}{l}\text { \# Events } \\
\text { total }\end{array}$ & $\begin{array}{c}\text { \# TBAD } \\
\text { detections }\end{array}$ & $\begin{array}{l}\text { \# TBAD only } \\
\text { (no FE) }\end{array}$ & $\begin{array}{l}\text { \# FE only } \\
\text { (no TBAD) }\end{array}$ & $\begin{array}{c}\text { \# Spotter } \\
\text { only call } \\
\text { (no TBAD) }\end{array}$ & Comments \\
\hline 2017.03 .07 & 12.17 & 22 & 20 & 6 & 0 & 2 & Most events of the run (1.8 per hour). \\
\hline 2017.03 .08 & 10.02 & 10 & 5 & 1 & 0 & 5 & Most spotter calls with no TBAD. \\
\hline 2017.03 .09 & 12.05 & 14 & 11 & 2 & 0 & 3 & \\
\hline 2017.03 .10 & 11.42 & 9 & 5 & 1 & 0 & 4 & \\
\hline 2017.03 .11 & 11.32 & 13 & 11 & 3 & 0 & 2 & \\
\hline 2017.03 .12 & 11.72 & 8 & 8 & 0 & 0 & 0 & \\
\hline 2017.03 .13 & 11.67 & 6 & 5 & 0 & 0 & 1 & \\
\hline 2017.03 .14 & 12.00 & 4 & 3 & 0 & 0 & 1 & Least events of the run ( 0.3 per hour). \\
\hline Totals & 92.4 & 86 & 68 & 13 & 0 & 18 & \\
\hline Average & 11.5 & 10.8 & 8.5 & 1.6 & 0.0 & 2.3 & \\
\hline Std Dev & 0.7 & 5.6 & 5.5 & 2.1 & 0.0 & 1.7 & \\
\hline
\end{tabular}

May 2017 Laser Run Summary

\begin{tabular}{|c|c|c|c|c|c|c|c|}
\hline UTC date & $\begin{array}{l}\text { Telescope } \\
\text { open time } \\
\text { (hh:mm) }\end{array}$ & $\begin{array}{c}\text { \# Events } \\
\text { total }\end{array}$ & $\begin{array}{c}\text { \# TBAD } \\
\text { detections }\end{array}$ & $\begin{array}{c}\text { \# TBAD only } \\
\text { (no FE) }\end{array}$ & $\begin{array}{l}\text { \# FE only } \\
\text { (no TBAD) }\end{array}$ & $\begin{array}{c}\text { \# Spotter } \\
\text { only call } \\
\text { (no TBAD) }\end{array}$ & Comments \\
\hline 2017.05 .02 & 10.02 & 5 & 4 & 1 & 0 & 1 & \\
\hline 2017.05 .03 & 9.53 & 11 & 9 & 1 & 0 & 2 & Most events of the run (1.15 per hour). \\
\hline 2017.05 .04 & 9.73 & 6 & 6 & 0 & 0 & 0 & \\
\hline 2017.05 .05 & 9.47 & 9 & 9 & 1 & 0 & 0 & \\
\hline 2017.05 .06 & 2.90 & 0 & 0 & 0 & 0 & 0 & Late opening for bad weather. \\
\hline 2017.05 .07 & 2.42 & 0 & 0 & 0 & 0 & 0 & Late opening for bad weather. \\
\hline 2017.05 .08 & 0.00 & $\mathrm{~N} / \mathrm{A}$ & $\mathrm{N} / \mathrm{A}$ & $\mathrm{N} / \mathrm{A}$ & $\mathrm{N} / \mathrm{A}$ & $\mathrm{N} / \mathrm{A}$ & Closed due to bad weather. \\
\hline 2017.05 .09 & 0.00 & $\mathrm{~N} / \mathrm{A}$ & $\mathrm{N} / \mathrm{A}$ & $\mathrm{N} / \mathrm{A}$ & $\mathrm{N} / \mathrm{A}$ & $\mathrm{N} / \mathrm{A}$ & Closed due to bad weather. \\
\hline 2017.05 .10 & 0.00 & $\mathrm{~N} / \mathrm{A}$ & $\mathrm{N} / \mathrm{A}$ & $\mathrm{N} / \mathrm{A}$ & $\mathrm{N} / \mathrm{A}$ & $\mathrm{N} / \mathrm{A}$ & Closed due to bad weather. \\
\hline Totals & 44.1 & 31 & 28 & 3 & 0 & 3 & \\
\hline Average & 7.3 & 5.2 & 4.7 & 0.5 & 0.0 & 0.5 & \\
\hline Std Dev & 3.6 & 4.5 & 4.1 & 0.5 & 0.0 & 0.8 & \\
\hline
\end{tabular}

\section{CONCLUSIONS}

After more than 3 years of safe laser operations, we are ready to transition to fully automatic aircraft detection for LGS operations.

Following on the steps of other observatories, we have been collecting and analyzing data to show that the use of TBAD is more reliable than the use of human spotters.

The implementation of TBAD-only operation will increase significantly the efficiency of the laser operations by an average of $20 \%$ for number of propagation closures, which does not take in account the reduction in closure time for every event. 


\section{ACKNOWLEDGEMENTS}

The authors want to acknowledge the work of the TBAD developers at UCSD (Tom Murphy and Bill Coles), for conceiving a simple but powerful and reliable system for the critical safety of laser operations.

Many thanks to Jackie Madsen from the Discovery Park in Safford, who served as our aircraft spotters coordinator. And to our spotters, for their enthusiasm and dedication, and for their hard work, especially during the coldest winter nights.

The LBT is an international collaboration among institutions in the United States, Italy and Germany. LBT Corporation partners are: The University of Arizona on behalf of the Arizona Board of Regents; Istituto Nazionale di Astrofisica, Italy; LBT Beteiligungsgesellschaft, Germany, representing the Max-Planck Society, The Leibniz Institute for Astrophysics Potsdam, and Heidelberg University; The Ohio State University, and The Research Corporation, on behalf of The University of Notre Dame, University of Minnesota and University of Virginia.

\section{REFERENCES}

[1] Rabien, S. et al, "ARGOS: the laser guide star system for the LBT”, Proc. SPIE 7736, Adaptive Optics Systems II, $77360 \mathrm{E}(2010)$.

[2] FAA Advisory Circular No 70-1 "Outdoor Laser Operations" (2004).

[3] ANSI Z136.6-2000 "Standard for Safe Use of Lasers Outdoors".

[4] Coles, W. et al, "A Radio System for Avoiding Illuminating Aircraft with a Laser Beam", PASP 124, 42-50, (2012).

[5] Stomski, P.; Murphy, T. and Campbell, R., "Recent developments in aircraft protection systems for laser guide star operations ", Proc. SPIE 8447, Adaptive Optics Systems III, 84474R (September 13, 2012).

[6] Murphy, Tom, "TBAD Performance Analysis at Apache Point" (APO internal memo).

[7] Rahmer, G. et al, "Aircraft avoidance for laser propagation at the Large Binocular Telescope Observatory. Life under a busy airspace", Proc. SPIE 9910, Observatory Operations: Strategies, Processes, and Systems VI, 99102G (15 July 2016).

[8] Federal Aviation Administration, "14 CFR Part 91 Automatic Dependent Surveillance - Broadcast (ADS-B) Out Performance Requirements To Support Air Traffic Control (ATC) Service; Final Rule” (May 28, 2010). 